\title{
ALOCAÇÃO E MODELAGEM DA BIOMASSA EM Dendrocalamus asper
}

\author{
Francelo Mognon ${ }^{1}$, Aurélio Lourenço Rodrigues ${ }^{1}$, Carlos Roberto Sanquetta ${ }^{2}$, Ana Paula Dalla Corte ${ }^{2}$, \\ Adalberto Brito de Novaes ${ }^{3}$, Christopher Thomas Blum ${ }^{2}$ \\ ${ }^{1}$ Universidade Federal do Paraná, Programa de Pós-Graduação em Engenharia Florestal, Curitiba, Paraná, Brasil - \\ mognonf@gmail.com; alourencorodrigues@gmail.com \\ ${ }^{2}$ Universidade Federal do Paraná, Departamento de Ciências Florestais, Curitiba, Paraná, Brasil - carlos_sanquetta@ hotmail.com; \\ anapaulacorte@gmail.com; ctblum.ufpr@gmail.com \\ ${ }^{3}$ Universidade Estadual do Sudoeste da Bahia, Departamento de Ciências Florestais, Vitória da Conquista, Bahia, Brasil - \\ adalberto.brito@globo.com
}

Recebido para publicação: 24/01/2014 - Aceito para publicação: 07/08/2014

O objetivo deste trabalho foi quantificar a biomassa seca total individual de plantas de bambu da espécie Dendrocalamus asper (Schult. \& Schult. f.) Backer ex K. Heyne, visando conhecer a sua distribuição nos diferentes compartimentos, bem como avaliar modelos de biomassa em função de variáveis biométricas das plantas. Foram avaliados 20 indivíduos, coletados em Bauru, SP. As plantas amostradas foram medidas, abatidas e pesadas. A maior fração da biomassa foi observada na parte aérea, com $86 \%$, sendo $64 \%$ para o compartimento colmo, $16 \%$ para os galhos e $6 \%$ para as folhas. Os rizomas representaram $14 \%$ da biomassa total. As variáveis biométricas (diâmetro à altura do peito - DAP, altura total - ht e diâmetro de colo - Dcolo) correlacionaram-se significativamente com as biomassas total e do colmo. O modelo que apresentou o melhor desempenho para a biomassa total teve como variável independente apenas o DAP, enquanto que para a biomassa dos colmos foi a variável combinada dap ${ }^{0,5}$ *Indap. Os ajustes para os demais compartimentos não geraram resultados satisfatórios, em função da baixa correlação entre as variáveis biométricas e suas biomassas. Concluiu-se que é possível expressar a biomassa seca total e do colmo do bambu por meio de modelos alométricos, porém o mesmo não se aplica aos demais compartimentos.

Palavras-chave: Bambu; fitomassa; modelos alométricos.

\begin{abstract}
Allocation and modeling of biomass of Dendrocalamus asper. The aim of this research was to quantify the total individual biomass of bamboo plants of the species Dendrocalamus asper (Schult. \& Schult. f.) Backer ex K. Heyne, in order to understand its distribution along different compartments, as well as evaluat biomass models according to biometric variables. Twenty individuals collected in Bauru, SP were evaluated. The plants were measured, cut and weighed. The aboveground biomass accounted for the major fraction, representing $86 \%$. The stem compartment represented $64 \%$ of total biomass, followed by the branches, with $16 \%$ and leaves, with $6 \%$. Rhizomes accounted for $14 \%$ of the total biomass. The biometric variables (diameter at breast height - $\mathrm{dbh}$, total height - ht, and collar diameter - dcollar) were significantly correlated with total and stem biomass. The model that revealed best performance for total biomass had only dap as independent variable, for the stems biomass the combined variable was dap $^{0,5 *}$ Indap. The adjustments for other compartments were not satisfactory due to low correlation between the biometric variables and their biomass. As conclusion, it is possible to express the total dry stem biomass and culm mass of bamboo using allometric models, however, the same does not apply to other compartments.

Keywords: Bamboo; phytomass; allometric models.
\end{abstract}

\section{INTRODUÇÃO}

A elevação nos níveis de dióxido de carbono na atmosfera e as consequentes mudanças climáticas de amplitude global têm aumentado consideravelmente a preocupação com o balanço do carbono nos

FLORESTA, Curitiba, PR, v. 45, n. 1, p. 1 - 10, jan. / mar. 2015.

Mognon, F. et al.

ISSN eletrônico 1982-4688 / ISSN impresso 0015-3826 
ecossistemas (BERT; DANJON, 2006). Tendo em vista a grande importância dos ecossistemas vegetais no ciclo do carbono, pesquisas científicas passaram a ser desenvolvidas com o intuito de melhorar a compreensão do papel de diferentes grupos de plantas ante o acúmulo de biomassa e carbono.

Para os estudos de carbono em florestas naturais ou plantadas, muitos autores utilizam a variável biomassa, a qual precisa ser determinada e/ou estimada de forma confiável (SANQUETTA, 2002). Uma das maneiras de se conhecer o estoque de carbono nos ecossistemas é por meio da determinação da biomassa e do desenvolvimento de modelos alométricos, fundamentais para a compreensão de aspectos ecológicos e evolutivos em espécies de plantas. Os modelos gerados a partir desses estudos podem ser ferramentas poderosas de predição em ecologia (PORTELA; SANTOS, 2003).

Os bambus têm sido reconhecidos como um grupo de espécies promissoras em função de sua rápida capacidade de sequestro de carbono atmosférico, entre outras possibilidades de utilização, dadas as suas excelentes propriedades físicas e mecânicas (BONILLA et al., 2010). Tais espécies compreendem um grupo de plantas pertencentes à família das gramíneas (Poaceae, subfamília Bambusoideae), representada por 1250 espécies, distribuídas em 75 gêneros, sendo que a maioria delas são de rápido crescimento, atingindo maturidade por volta dos 5 anos (SCURLOCK et al., 2000).

Os bambus estão presentes, em sua maioria, nas regiões tropicais, podendo ocorrer naturalmente em zonas subtropicais e temperadas em todos os continentes, com exceção da Europa, entre latitudes de $46 \mathrm{~N}$ a $47 \mathrm{~S}$, em altitudes que vão do nível do mar até aproximadamente $4.000 \mathrm{~m}$ (WILLIAMS et al., 1994). Estima-se que no mínimo 40 milhões de ha da superfície terrestre sejam compostos por florestas de bambus, representando aproximadamente $1 \%$ da cobertura florestal mundial (LOBOVIKOV et al., 2007).

Ao contrário da maioria das gramíneas, mas semelhantemente às árvores, os bambus apresentam o sistema fotossintético $\mathrm{C}_{3}$ (LARCHER, 2000; DÜKING et al., 2011). Dessa forma, na ausência de fatores limitantes, são capazes de aumentar o seu crescimento em biomassa em resposta a uma atmosfera enriquecida com $\mathrm{CO}_{2}$ (LOBOVIKOV et al., 2012).

Entre as espécies de bambu com grande potencial em razão de seu rápido crescimento e expressiva biomassa, pode-se citar o bambu-gigante (Dendrocalamus asper (Schult. \& Schult. f.) Backer ex K. Heyne). Natural do sudeste asiático, esse bambu simpodial apresenta colmos com altura de 20 a $30 \mathrm{~m}$ e diâmetro que pode variar de 8 a $20 \mathrm{~cm}$. Desenvolve-se em regiões tropicais úmidas e em regiões subtropicais, preferindo solos ricos (PEREIRA; BERALDO, 2007).

Apesar de sua potencialidade, estudos sobre a biomassa de bambus são escassos e limitados, em sua maioria, a povoamentos naturais, com poucas referências a plantios. Sendo assim, trabalhos que tratem desse assunto são de especial relevância, especialmente no que se refere ao estudo das relações alométricas para a estimativa da biomassa, visto que é um pressuposto básico para estudo da fixação e balanço de carbono (NATH et al., 2009). No Brasil, estudos sobre essa temática são raros. Diante disso, o presente trabalho tem como objetivo quantificar a distribuição da biomassa seca nos compartimentos da espécie $D$. asper, explorar as relações alométricas nessa espécie e ajustar equações para a aplicação em estudos de produtividade primária, como forma de potencializar o uso dessa espécie de bambu no Brasil.

\section{MATERIAL E MÉTODOS}

Os dados são oriundos de um cultivo conduzido na área Experimental Agrícola da Universidade Estadual Paulista (UNESP), Campus de Bauru, SP. A área está contida no sistema geodésico regional SAD 69, UTM fuso 22K, com coordenadas centrais, X: 703155m e Y: $7526389 \mathrm{~m}$, conforme figura 1 . A altitude regional é próxima dos 560 metros. O clima do município de Bauru é classificado como úmido e mesotérmico, com temperatura média anual de $22,7^{\circ} \mathrm{C}$ (FIGUEIREDO; PAES, 2010).

Neste estudo, avaliou-se a biomassa de 20 indivíduos da espécie $D$. asper, com diferentes diâmetros. Selecionaram-se aleatoriamente touceiras de bambu, dentre as quais foram coletados os indivíduos para medição e determinação de biomassa seca.

Os indivíduos foram cortados na base do colmo e pesados empregando-se o método destrutivo, utilizando-se para as pesagens o método da simples separação (SANQUETTA, 2002), considerando os seguintes compartimentos da biomassa verde: colmo, galho, folhagem e rizoma (foram retirados por meio de trincheiras escavadas em torno do indivíduo). As variáveis biométricas circunferência à altura do peito (CAP), circunferência do colo (Ccolo) e altura total (ht) foram medidas após o abate dos indivíduos, obtendo-se posteriormente o diâmetro à altura do peito (DAP) e o diâmetro do colo (Dcolo). Todo o 
material foi pesado com uso de balança analógica e amostras de aproximadamente $500 \mathrm{~g}$ da biomassa fresca de cada compartimento foram retiradas, embaladas em sacos plásticos e encaminhadas ao Centro de Excelência em Pesquisas sobre Fixação de Carbono na Biomassa (Biofix), da Universidade Federal do Paraná (UFPR), onde foram submetidas ao processo de secagem em estufa com circulação de ar forçada, a uma temperatura de $70{ }^{\circ} \mathrm{C}$ até atingir peso constante.

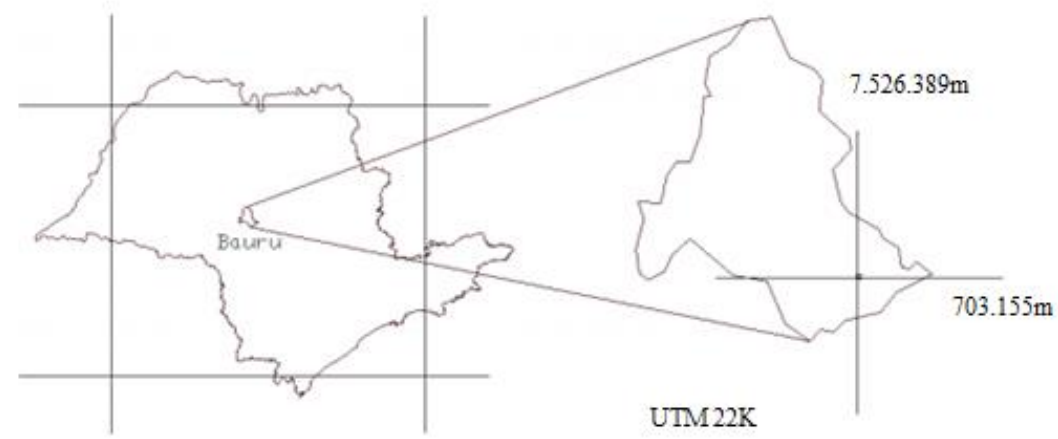

Figura 1. Localização da área de estudo (adaptado de Carboni, 2011).

Figure 1. Location of the focused area (adapted from Carboni, 2011).

Foram realizadas análises de correlação linear simples de todas as variáveis de medição das plantas abatidas com suas respectivas biomassas secas, considerando cada um dos compartimentos, e, finalmente, a biomassa total, com o objetivo de avaliar as relações alométricas da espécie.

Para a estimativa indireta da biomassa seca total das plantas, foram testados modelos matemáticos usualmente empregados para estimativa dessa variável (Tabela 1). Os ajustes foram efetuados por meio do método dos mínimos quadrados.

Tabela 1. Modelos matemáticos testados para a estimativa da biomassa seca total (Bs) de Dendrocalamus asper.

Table 1. Fitted equations for dry weight (Bs) estimating of Dendrocalamus asper.

\begin{tabular}{|c|c|}
\hline $\mathbf{N}^{0}$ & Modelo \\
\hline 1 & $\mathrm{Bs}=\beta_{0}+\beta_{1} \mathrm{ht}$ \\
\hline 2 & $\mathrm{Bs}=\beta_{0}+\beta_{1}$ dap \\
\hline 3 & $\mathrm{Bs}=\beta_{0}+\beta_{1} \mathrm{dap}^{2}$ \\
\hline 4 & $\mathrm{Bs}=\beta_{0}+\beta_{1} \mathrm{dap} * \mathrm{ht}^{2}$ \\
\hline 5 & $\mathrm{Bs}=\beta_{0}+\beta_{1} \mathrm{dap}+\beta_{2} \mathrm{~h}$ \\
\hline 6 & $\mathrm{Bs}=\beta_{0}+\beta_{1}$ dcolo $+\beta_{2} \mathrm{dap}^{2}+\beta_{3}\left(\mathrm{dap}^{*} \mathrm{ht}^{2}\right)$ \\
\hline 7 & $\ln \mathrm{Bs}=\beta_{0}+\beta_{1} \operatorname{lnht}^{2}$ \\
\hline 8 & $\ln \mathrm{Bs}=\beta_{0}+\beta_{1} \ln \mathrm{dap}$ \\
\hline 9 & $\ln \mathrm{Bs}=\beta_{0}+\beta_{1} \operatorname{lndcolo}+\beta_{2} \ln \mathrm{dap}^{2}+\beta_{3} \ln \left(\mathrm{dap}^{*} \mathrm{ht}^{2}\right)$ \\
\hline 10 & $\mathrm{Bs}=\beta_{0}+\beta_{1}\left(\mathrm{dap}^{0.5 * \ln } \mathrm{dap}\right)$ \\
\hline
\end{tabular}

A avaliação do desempenho dos modelos foi feita por meio do coeficiente de determinação ajustado ( $\mathrm{R}^{2}$ aj.) e erro padrão da estimativa (Syx\%), assim como pela análise gráfica dos resíduos.

\section{RESULTADOS E DISCUSSÃO}

\section{Distribuição de biomassa e correlação com as variáveis biométricas}

A figura 2 ilustra a distribuição de biomassa nos respectivos compartimentos das plantas. Podese verificar que a maior fração de biomassa está concentrada na parte aérea, com destaque para o compartimento colmo, com $64 \%$, sendo também computados os galhos secos, os quais contribuíram com $0,3 \%$ da biomassa total. Tais resultados corroboram Marenco e Lopes (2005), que reportam que as espécies de bambu apresentam maior concentração de seus assimilados na parte aérea. 


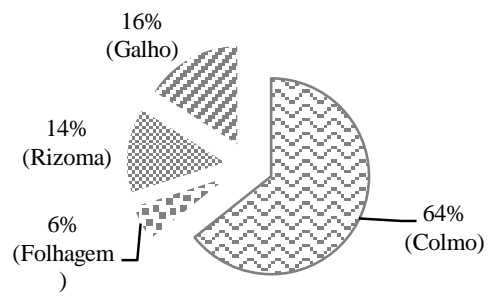

Figura 2. Distribuição da biomassa em compartimentos de plantas de Dendrocalamus asper. Figure 2. Biomass distribution in different compartments of Dendrocalamus asper.

Os galhos vivos representaram 16\% da biomassa, seguidos pelos rizomas, com 14\%, e folhas, com 6\%. Shanmughavel e Francis (1996), trabalhando com plantas de Bambusa bamboos (Linn.), encontraram $80-85 \%$ de sua biomassa distribuída nos colmos, $15-20 \%$ nos galhos e apenas $1 \%$ nas folhas. Já em plantas da espécie Yashania alpinia (K.Schum.) W.C.Lin, aos três anos de idade, Embaye et al. (2005) reportaram uma distribuição de biomassa na ordem de $80 \%, 13 \%$ e 5\%, respectivamente, para colmo, raízes e folhas, resultados próximos aos observados no presente estudo.

As correlações entre as variáveis biométricas e a biomassa dos compartimentos do bambu foram fortes e significativas entre DAP e biomassa dos colmos, bem como com a biomassa total. O diâmetro de colo correlacionou-se bem com a biomassa dos colmos e a total. A altura total também apresentou correlações satisfatórias com a biomassa do colmo e biomassa total (Tabela 2). Por outro lado, a correlação entre a biomassa dos demais compartimentos com as variáveis biométricas não foram significativas. Tais resultados devem-se à maior variabilidade natural desses compartimentos e ao fato de que as variáveis explicativas são obtidas diretamente sobre o colmo, aumentando a correlação da biomassa seca do mesmo com as variáveis independentes, enquanto que para os demais compartimentos a relação com as variáveis biométricas é apenas indireta.

Tabela 2. Análise de correlação entre as variáveis biométricas de Dendrocalamus asper.

Table 2. Correlation analysis between biometric variables of Dendrocalamus asper.

\begin{tabular}{lcccccccc}
\hline & \multirow{2}{*}{ ht (m) } & \multirow{2}{*}{ Dcolo $(\mathbf{c m})$} & \multirow{2}{*}{ DAP $(\mathbf{c m})$} & \multicolumn{5}{c}{ Biomassa seca (Bs) } \\
\cline { 5 - 8 } & & & Colmo & Folhagem & Rizoma & Galho & Total \\
\hline ht $(\mathrm{m})$ & 1,000 & & & & & & & \\
Dcolo $(\mathrm{cm})$ & $0,724^{*}$ & 1,000 & & & & & & \\
DAP $(\mathrm{cm})$ & $0,818^{*}$ & $0,882^{*}$ & 1,000 & & & & & \\
Bs colmo & $0,794^{*}$ & $0,783^{*}$ & $0,865^{*}$ & 1,000 & & & & \\
Bs folhagem & $-0,292$ & 0,080 & $-0,027$ & 0,043 & 1,000 & & & \\
Bs rizoma & 0,235 & 0,251 & 0,351 & $0,540^{*}$ & 0,308 & 1,000 & & \\
Bs galho & 0,089 & 0,299 & 0,291 & 0,274 & $0,676^{*}$ & 0,288 & 1,000 & \\
Bs total & $0,628^{*}$ & $0,710^{*}$ & $0,781^{*}$ & $0,917^{*}$ & 0,359 & $0,698^{*}$ & $0,590^{*}$ & 1,000 \\
\hline
\end{tabular}

* Correlações significativas $(\mathrm{p}<0,05)$.

Na figura 3 observam-se estreitas correlações e reduzida dispersão da biomassa seca total e do compartimento do colmo com as variáveis biométricas DAP, Dcolo e ht. Justificam-se tais correlações em função da elevada participação da biomassa do colmo em relação à biomassa total.

Ressalte-se que a variável DAP é de fácil medição e, com base nos resultados de correlação, tem alto poder preditivo da biomassa seca total e relativa ao colmo. Sendo assim, esses resultados apontam para uma provável explicação das biomassas total e do colmo em função das variáveis biométricas DAP, Dcolo e ht, possibilitando o desenvolvimento de modelos alométricos, visando estimar as biomassas em função das variáveis de fácil obtenção.

\section{Teste de modelos alométricos}

Com base na análise de correlação entre as variáveis biométricas e a biomassa do bambu, ajustaram-se equações apenas para a biomassa total e do colmo, visto que a biomassa dos demais compartimentos não apresentou correlações significativas (Tabelas 3 e 4). 

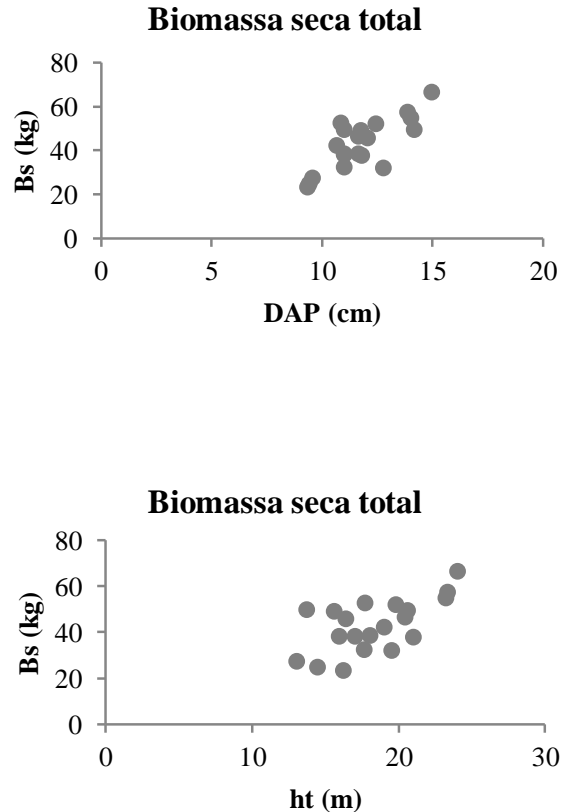

Biomassa seca do colmo

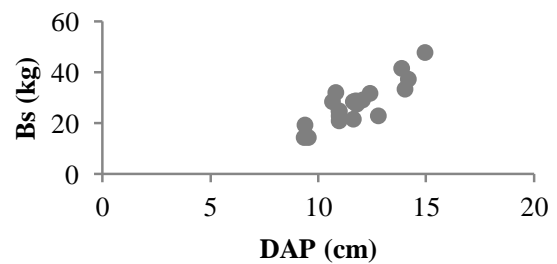

Biomassa seca total

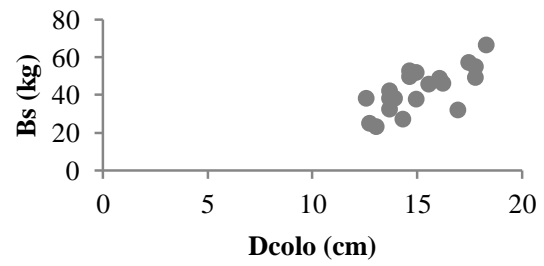

Biomassa seca do colmo

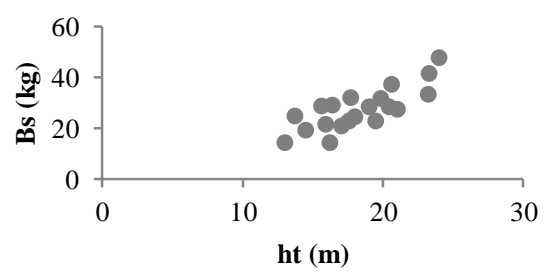

Biomassa seca do colmo

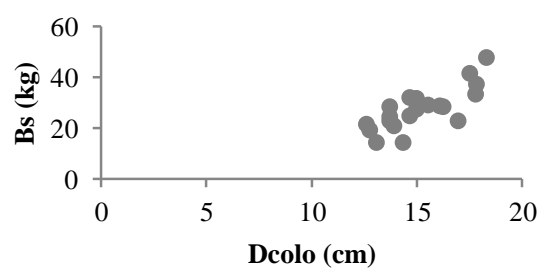

Figura 3. Relação entre variáveis biométricas e de biomassa seca em plantas de Dendrocalamus asper. Figure 3. Relation between biometric variables and dry biomass in Dendrocalamus asper.

As equações ajustadas para a biomassa total apresentaram resultados satisfatórios em relação às estatísticas de precisão, com valores de $\mathrm{R}^{2}$ ajustado próximos a 0,60 e Syx $\%$ abaixo de $20 \%$ (Tabela 3). Das equações ajustadas, as que apresentaram melhores estatísticas foram 2, 3 e 8 . Essas equações são de simples entrada e apresentam o DAP (original ou transformado) como variável independente. Na figura 4 (coluna a) estão apresentados os resíduos das melhores equações para estimativas da biomassa seca total.

Tabela 3. Estatísticas de ajuste de equações de biomassa seca total para Dendrocalamus asper.

Table 3. Adjustment statistics of total dry biomass equations for Dendrocalamus asper.

\begin{tabular}{lcccccc}
\hline Modelo & $\boldsymbol{\beta}_{\boldsymbol{0}}$ & $\boldsymbol{\beta}_{\boldsymbol{1}}$ & $\boldsymbol{\beta}_{\mathbf{2}}$ & $\boldsymbol{\beta}_{\mathbf{3}}$ & $\mathbf{R}^{\mathbf{2}}$ ajustado & Syx \% \\
\hline 1 & 1,29628 & 2,28201 & & & 0,36 & 21,33 \\
2 & $-23,25707$ & 5,65645 & & & 0,59 & 17,12 \\
3 & 10,65857 & 0,23170 & & & 0,57 & 17,41 \\
4 & 25,78702 & 0,00413 & & & 0,47 & 19,40 \\
5 & $-23,35328$ & 5,85042 & $-0,11896$ & & 0,56 & 17,61 \\
6 & 3,60545 & 0,73139 & 0,20555 & $-0,00009$ & 0,52 & 18,40 \\
7 & 2,31329 & 0,16824 & & & 0,36 & 21,29 \\
8 & $-0,35895$ & 1,66516 & & & 0,57 & 17,43 \\
9 & 1,75746 & 0,12606 & 0,35473 & $-0,06239$ & 0,51 & 18,60 \\
10 & 1,92972 & 0,21355 & & & 0,56 & 17,65 \\
\hline
\end{tabular}

FLORESTA, Curitiba, PR, v. 45, n. 1, p. 1 - 10, jan. / mar. 2015. 
Tabela 4. Estatísticas de ajuste de equações de biomassa seca do colmo para Dendrocalamus asper.

Table 4. Adjustment statistics of total dry biomass equations for Dendrocalamus asper.

\begin{tabular}{lcccccc}
\hline Equação & $\boldsymbol{\beta}_{\boldsymbol{0}}$ & $\boldsymbol{\beta}_{\boldsymbol{1}}$ & $\boldsymbol{\beta}_{\mathbf{2}}$ & $\boldsymbol{\beta}_{\mathbf{3}}$ & $\mathbf{R}^{\mathbf{2}}$ ajustado & Syx\% \\
\hline 1 & $-10,92783$ & 2,10624 & & & 0,61 & 18,94 \\
2 & $-26,00229$ & 4,57398 & & & 0,73 & 15,62 \\
3 & 1,05172 & 0,19001 & & & 0,74 & 15,47 \\
4 & 10,80322 & 0,00634 & & & 0,72 & 16,17 \\
5 & $-25,44223$ & 3,44490 & 0,69248 & & 0,76 & 14,91 \\
6 & 2,77087 & 0,21734 & 0,10728 & 0,00157 & 0,73 & 15,65 \\
7 & 1,29031 & 0,23631 & & & 0,63 & 18,58 \\
8 & $-1,56546$ & 1,97305 & & & 0,74 & 15,50 \\
9 & $-0,27646$ & 0,10637 & 0,25021 & 0,21274 & 0,73 & 15,75 \\
10 & 1,12544 & 0,25553 & & & 0,74 & 15,47 \\
\hline
\end{tabular}

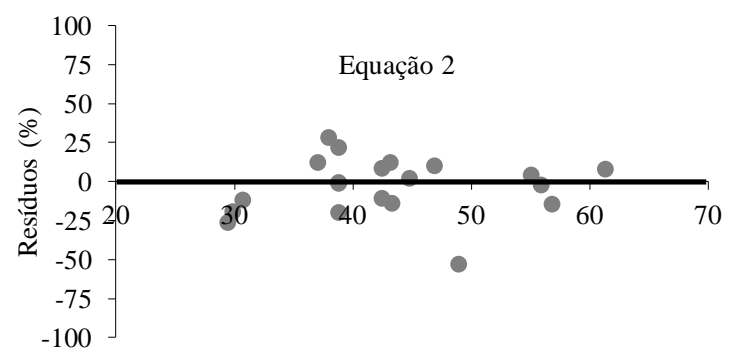

Biomassa seca estimada $(\mathrm{kg})$

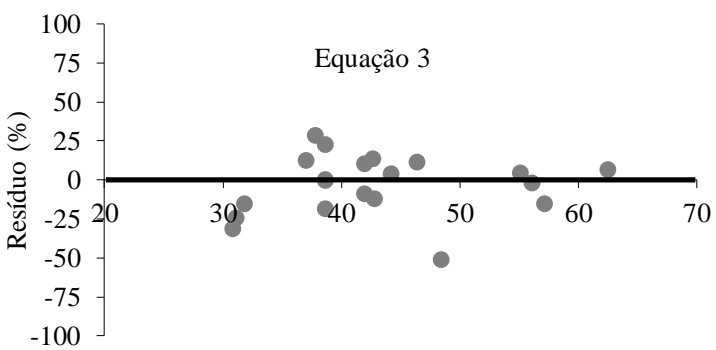

Biomassa seca estimada $(\mathrm{kg})$

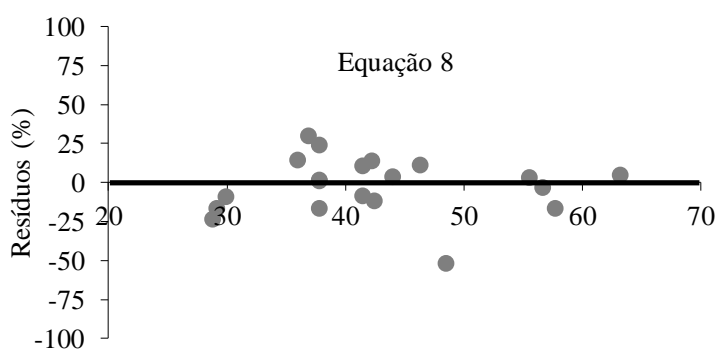

Biomassa seca estimada $(\mathrm{kg})$

(a)

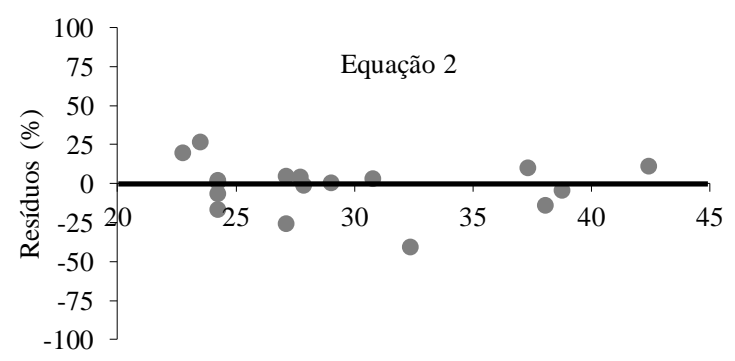

Biomassa seca do colmo estimada $(\mathrm{kg})$
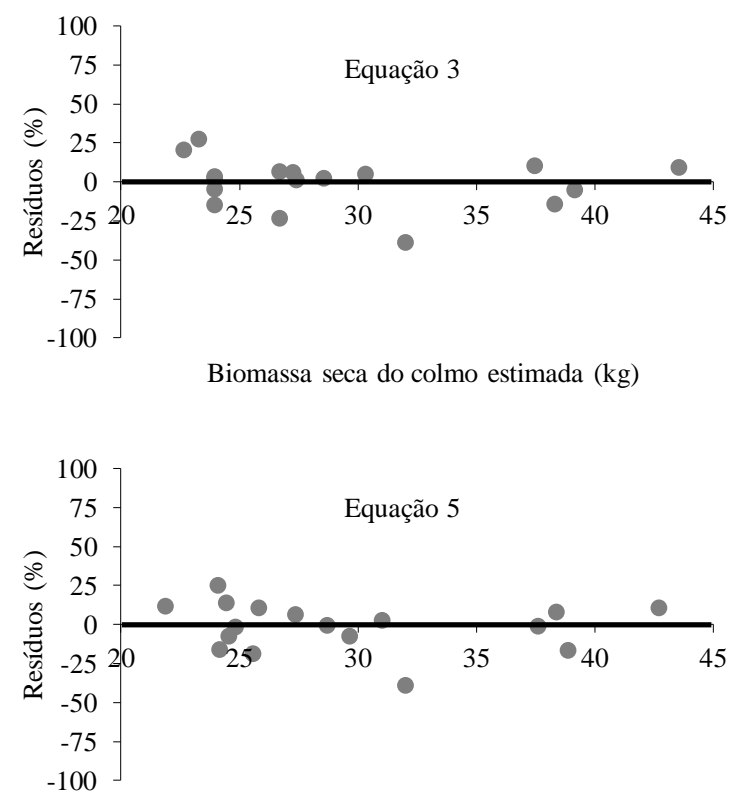

Biomassa seca do colmo estimada $(\mathrm{kg})$

(b)

Figura 4. Distribuição de resíduos correspondentes aos melhores ajustes para as equações: (a) Biomassa seca total; e (b) Biomassa seca do colmo.

Figure 4. Graphical distribution of residuals corresponding to adjustments of the equations: (a) Total biomass, and (b) Biomass of stems. 
As equações ajustadas para o compartimento do colmo apresentaram desempenho superior em relação às estatísticas de precisão, quando comparadas com os resultados obtidos para o total, com valores de $\mathrm{R}^{2}$ ajustado maiores que 0,70 e Syx $\%$ de aproximadamente $15 \%$ (Tabela 4). Trabalhando nessa mesma linha de pesquisa e com a espécie Dendrocalamus strictus (Roxb.) Nees, Singh e Singh (1999) observaram uma tendência similar no ajuste de equações em função do DAP para estimativa de biomassa de colmo, encontrando $\mathrm{R}^{2}$ equivalente a 0,93 . O melhor ajuste obtido pelos autores em relação ao presente estudo pode ser atribuído às características do experimento, visto que o mesmo apresentava espaçamento menor, além de as touceiras serem mais jovens que as deste estudo, o que pode resultar em uma maior homogeneidade dos plantios, propiciando melhores ajustes.

À luz dessas constatações, pode-se observar que os ajustes efetuados para o compartimento do colmo mostraram-se mais eficientes quando comparados com os da biomassa seca total. Das equações ajustadas, as que apresentaram melhores estatísticas foram 2, 3, 5, 8 e 10, com resultados semelhantes. Apesar de a equação 5 apresentar resultados de ajustes ligeiramente maiores, ela requer dupla entrada de variáveis independentes (DAP e ht). Quanto às demais equações, apenas a variável DAP (original ou transformada) é requerida como variável explanatória.

A figura 3 apresenta a distribuição dos resíduos para as equações de melhores ajustes.

Trabalhando com a espécie Guadua weberbaueri Pilger, Torezan e Silveira (2000) utilizaram DAP e altura do colmo para estimativa de biomassa, encontrando um $\mathrm{R}^{2}$ de 0,74 , considerada uma boa estimativa. Esses mesmos autores ainda encontraram um resultado mais favorável, com um $\mathrm{R}^{2}$ de 0,75 , utilizando uma equação em função apenas do DAP. Outros autores, como Shanmughavel e Francis (1996), encontraram um $\mathrm{R}^{2}$ de 0,83 para estimativa de biomassa total em plantas de Bambusa bamboos. Esses autores ressaltam as dificuldades do uso de variáveis como a altura em determinadas equações, tendo em vista as dificuldades de sua mensuração, daí a preferência pelo uso apenas do DAP, pela sua maior praticidade e menor tendência ao erro. Em se tratando do presente estudo, observou-se a mesma analogia, tendo em vista que os ajustes, utilizando ou não a altura, foram similares.

A distribuição dos resíduos dos melhores ajustes para a espécie em estudo, correspondente a biomassas do colmo e total, pode ser verificada na figura 3. Nela, pode-se observar que a equação 2 $(\mathrm{Bs}=-23,25707+5,65645 *$ dap $)$ apresentou melhor distribuição dos resíduos para a biomassa seca total, enquanto que a equação $5(\mathrm{Bs}=-2544223+3,44490 *$ dap $+0,69248 * \mathrm{ht})$ apresentou a melhor distribuição dos resíduos para a estimativa da biomassa seca do colmo. Para a equação da biomassa relativa ao colmo, os resíduos variaram de -42 a $24 \%$, demonstrando uma maior homogeneidade quando comparado com a equação para a estimativa de biomassa total, onde os resíduos variaram de -53 a $28 \%$.

\section{Cenário de produção de biomassa e comparação com outras espécies}

Visando avaliar o potencial de acúmulo de biomassa da espécie em plantios, simulou-se um cenário com base nos dados de Pereira e Garbino (2003), que estudaram o desenvolvimento de D. giganteus após 8 anos de plantio manejado com espaçamento de 8 x $8 \mathrm{~m}$ (156 moitas $/ \mathrm{ha})$, em Bauru, SP. A produtividade média observada pelos autores foi de 1.462 novos colmos/ha/ano. Supondose um plantio com as mesmas características encontradas pelos autores acima, em um período de sete anos, estima-se uma densidade de 10.234 colmos/ha.

Aplicando-se a equação $2\left(\mathrm{Bs}=-23,25707+5,65645^{*}\right.$ dap $)$ e utilizando o cenário citado, bem como os valores médios de DAP $(11,7 \mathrm{~cm})$, obteve-se a estimativa de biomassa seca total de 439,28 tha ${ }^{-1}$. Entretanto, essa equação apresentou resíduos variando de -53 a $28 \%$, conforme a figura 3a. Visando verificar a influência dos valores extremos sobre as estimativas, comparou-se a equação mencionada com outra ajustada com base nos mesmos dados, exceto o ponto mais discrepante (-53\%). Observou-se então uma distribuição dos resíduos mais homogênea (-25 a 27\%), porém essa nova equação gerou superestimativa significativa dos valores de biomassa total seca, conforme teste $\mathrm{t}$ a $95 \%$ de probabilidade. Dessa forma, considerando que o ponto excluído trata-se de um dado real e sua exclusão é arbitrária, optou-se por manter a equação original, considerando coerente o cenário estimado por meio dela.

Em virtude da incipiência dos estudos com $D$. asper, não foram encontradas referências consistentes na literatura a respeito da produção de biomassa seca por essa espécie. Portanto, comparações foram feitas com outras espécies de bambus. Nath et al. (2009), avaliando a biomassa de três espécies de bambus (Bambusa cacharensis R. Majumder, Bambusa vulgaris Schrad. e Bambusa balcooa Roxb.) cultivadas em plantios mistos na Índia, observaram valores de 121.51 t.ha $^{-1}$ de biomassa

FLORESTA, Curitiba, PR, v. 45, n. 1, p. 1 - 10, jan. / mar. 2015. 
seca. Embaye et al. (2005) observaram produção total de biomassa aérea de 110 t.ha $^{-1}$ em cultivos no sudoeste da Etiópia. Shanmughavel e Francis (1996), estudando a produção de biomassa de Bambusa bamboos, obtiveram valor de 286 t.ha $^{-1}$, considerando moitas aos seis anos de idade. Em cultivos da espécie de bambu Phyllostachys bambusoides Madake no Japão, Isagi et al. (1993) observaram produção total de biomassa de 131 t.ha ${ }^{-1}$, considerando moitas entre 16 e 22 anos. Singh e Singh (1999) relataram produção total de biomassa em cultivos de bambu variando de 46,9 t.ha $^{-1}$ a 74,7 t.ha $^{-1}$ aos 3 e 7 anos, respectivamente.

Ao considerar espécies arbóreas, como o eucalipto, Santana (2008) obteve valores de biomassa aérea variando de 58,4 t.ha ${ }^{-1}$ a 212,1 t.ha $^{-1}$ aos sete anos, na região Sudeste do Brasil. Gatto et al. (2011), também avaliando os estoques de biomassa em plantios de eucalipto, constataram variação de 115,83 a 168,26 t.ha $^{-1}$ de biomassa aérea. Considerando a biomassa aérea de D. asper (descontando-se os 14\% de rizomas, como observado no presente estudo), obtém-se 377,78 t.ha $^{-1}$, valor ainda superior ao da cultura florestal de maior destaque no Brasil. Tendo em vista os valores obtidos pelos autores citados, observamos que as estimativas de biomassa de $D$. asper são consideravelmente superiores, podendo indicar uma boa capacidade produtiva para essa espécie.

\section{CONCLUSÕES}

- Concluiu-se que é possível expressar a biomassa seca total e do colmo do bambu por meio de modelos alométricos, porém o mesmo não se aplica aos demais compartimentos.

- A biomassa seca total e do colmo correlacionaram-se fortemente com as variáveis biométricas DAP, Dcolo e ht.

- Para a estimativa da biomassa seca total, modelos apenas com o DAP como variável independente apresentaram melhor desempenho. Já para a biomassa seca do colmo, o modelo com DAP e altura total foi o mais satisfatório. Contudo, em função da dificuldade da obtenção da altura total, a estimativa da biomassa seca do colmo pode ser expressa por meio de modelos apenas com o DAP como variável independente sem maiores prejuízo na qualidade das estimativas.

\section{REFERÊNCIAS}

BERT, D.; DANJON, F. Carbon concentration variations in the roots, stem and crown of mature Pinus pinaster (Ait.). Forest Ecology and Management, v. 222, p. 279 - 295, 2006.

BONILLA, S. H.; GUARNETTI, R. L.; ALMEIDA, C. M. V. B.; GIANNETTI, B. F. Sustainability assessment of a giant bamboo plantation in Brazil: exploring the influence of labour, time and space. Journal of Cleaner Production, v. 18, p. 83 - 91, 2010.

CARBONI, M. Dinâmica estrutural e reprodutiva da vegetação lenhosa de uma floresta paludosa em Bauru/SP. 156 f. Tese (Doutorado em Ciências) - Escola Superior de Agricultura "Luiz de Queiroz", Piracicaba, 2011.

DÜKING, R.; GIELIS, J.; WALTER, L. Carbon flux and carbon stock in a bamboo stand and their relevance for mitigating climate change. Journal of the American Bamboo Society, v. 24, p. 1 - 7, 2011.

EMBAYE, K.; WEIHA, M.; LEDINC, S.; CHRISTERSSONA, L. Biomass and nutrient distribution in a highland bamboo forest in southwest Ethiopia: implications for management. Forest Ecologyand Management, v. 204, n. 1, p. 159 - 169, 2005.

FIGUEIREDO, J. C.; PAZ, R. S. Nova classificação climática e o aspecto climático da cidade de Bauru/São Paulo. In: CONGRESSO BRASILEIRO DE METEOROLOGIA, 15. 2010, Belém. Anais... Bauru, 2010.

GATTO, A.; BARROS, N. F.; NOVAIS, R. F.; SILVA, I. R.; LEITE, H. G.; VILLANI, E. M. A. Estoque de carbono na biomassa de plantações de eucalipto na região centro-leste do estado de Minas Gerais. Revista Árvore, v. 35, n. 4, p. 895 - 905, 2011.

ISAGI, Y.; KAWAHARA, T.; KAMO, K. Biomass and net production in a bamboo Phyllostachys bambusoides stand. Ecological Research, v. 8, p. 123 - 133, 1993. 
LARCHER, W. Ecofisiologia vegetal. São Carlos: Rima Artes e Textos, 2000. 531 p.

LOBOVIKOV, M.; PAUDEL, S.; PIAZZA, M.; REN, H.; WU, J. World bamboo resources. Rome: FAO, 2007. $73 \mathrm{p}$.

LOBOVIKOV, M.; SCHOENE, D.; YPING, L. Bamboo in climate change and rural livelihoods. Mitigation and Adaptation Strategies for Global Change, v. 17, p. 261 - 276, 2012.

MARENCO, R. A.; LOPES, N. F. Fisiologia Vegetal: Fotossíntese, respiração, relações hídricas e nutrição mineral. Viçosa: Editora UFV, 2005. 451 p.

NATH, A. J.; DAS, G.; DAS, A. K. Above ground standing biomass and carbon storage in village bamboos in North East India. Biomass and Bioenergy, v. 33, p. 1188 - 1196, 2009.

PEREIRA, M. A. R.; GARBINO, L. V. Desenvolvimento e produção do bambu-gigante (Dendrocalamus giganteus) cultivado na UNESP/Campus de Bauru, SP, com vistas à sua utilização na engenharia agrícola. In: CONGRESSO BRASILEIRO DE ENGENHARIA AGRÍCOLA, 32., 2003, Goiânia. Anais... Goiânia, 2003. p. 4.

PEREIRA, M. A. R.; BERALDO, A. L. Bambu de corpo e alma. Bauru: Canal 6, 2007. 240 p.

SANQUETTA, C. R. Métodos de determinação de biomassa florestal. In: SANQUETTA, C. R.; WATZLAWICK, L. F.; BALBINOT, R.; ZILLIOTTO, M. A.; GOMES, F. S. As florestas e o carbono. Curitiba: UFPR, p. 119 - 140, 2002.

SANTANA, R. C.; BARROS, N. F.; LEITE, H. G.; COMERFORD, N. B.; NOVAIS, R. F. Estimativas de biomassa de plantios de eucalipto no Brasil. Revista Árvore, v. 32, n. 4, p. 697 - 706, 2008.

SCURLOCK, J. M. O.; DAYTONB, D. C.; HAMESB, B. Bamboo: an overlooked biomass resource? Biomass and Bioenergy, v. 19, n. 3, p. 229 - 244, 2000.

SHANMUGHAVEL, P.; FRANCIS, K. Above ground biomass production and nutrient distribution in growing bamboo (Bambusa bamboos L.). Biomass and Bioenergy, v. 10, n. 2, p. 383 - 391, 1996.

SINGH, A. N.; SINGH, J. S. Biomass, net primary production and impact of bamboo plantation on soil redevelopment in a dry tropical region. Forest Ecology and Management, v. 119, n. 2, p. 195 - 207, 1999.

TOREZAN, J. M. D.; SILVEIRA, M. The biomass of bamboo (Guadua weberbaueri Pilger) in open forest of the southwestern Amazon. Ecotropica, v. 2. n. 6, p. 71 - 76, 2000.

WILLIAMS, J. T.; DRANSFIELD, J.; GANAPATHY, P. M.; LIESE, W.; NOR, S. M.; SASTRY, C. B. Research needs for bamboo and rattan to the year 2000. Beijing: INBAR, 1994. $86 \mathrm{p}$. 
FLORESTA, Curitiba, PR, v. 45, n. 1, p. 1 - 10, jan. / mar. 2015. Mognon, F. et al. 